\title{
A role for tetraspanin proteins in regulating fusion induced by Burkholderia thailandensis
}

\author{
Atiga Elgawidi ${ }^{1}$ Muslim Idan Mohsin ${ }^{1,3} \cdot{\text { Fawwaz } \text { Ali }^{1,4} \cdot \text { Amyleigh Watts }^{1} \cdot \text { Peter N. Monk }}^{2} \cdot$ Mark S. Thomas ${ }^{2}$. \\ Lynda J. Partridge ${ }^{1}$
}

Received: 31 January 2020 / Accepted: 23 March 2020 / Published online: 6 April 2020

(c) The Author(s) 2020

\begin{abstract}
Burkholderia pseudomallei is the causative agent of melioidosis, a disease with high morbidity that is endemic in South East Asia and northern Australia. An unusual feature of the bacterium is its ability to induce multinucleated giant cell formation (MNGC), which appears to be related to bacterial pathogenicity. The mechanism of MNGC formation is not fully understood, but host cell factors as well as known bacterial virulence determinants are likely to contribute. Since members of the tetraspanin family of membrane proteins are involved in various types of cell:cell fusion, their role in MNGC formation induced by Burkholderia thailandensis, a mildly pathogenic species closely related to B. pseudomallei, was investigated. The effect of antibodies to tetraspanins CD9, CD81, and CD63 in MNGC formation induced by $B$. thailandensis in infected mouse J774.2 and RAW macrophage cell lines was assessed along with that of recombinant proteins corresponding to the large extracellular domain (EC2) of the tetraspanins. B. thailandensis-induced fusion was also examined in macrophages derived from CD9 null and corresponding WT mice, and in J774.2 macrophages over-expressing CD9. Antibodies to CD9 and CD81 promoted MNGC formation induced by $B$. thailandensis, whereas EC2 proteins of CD9, CD81, and CD63 inhibited MNGC formation. Enhanced MNGC formation was observed in CD9 null macrophages, whereas a decrease in MNGC formation was associated with overexpression of CD9. Overall our findings show that tetraspanins are involved in MNGC formation induced by B. thailandensis and by implication, B. pseudomallei, with CD9 and CD81 acting as negative regulators of this process.
\end{abstract}

Keywords Burkholderia · Melioidosis · Tetraspanin · Multinucleated giant cell · CD9 · Cell:cell fusion

Edited by Luise Florin.

This article is part of the Special Issue on Tetraspanins in Infections and Immunity.

Lynda J. Partridge

1.partridge@sheffield.ac.uk

1 Department of Molecular Biology and Biotechnology, University of Sheffield, Sheffield S10 2TN, UK

2 Department of Infection, Immunity and Cardiovascular Disease, Medical School, University of Sheffield, Sheffield S10 2RX, UK

3 Present Address: Department of Pathological Analyses, University of Kufa, Kufa, Iraq

4 Present Address: Mosul Technical Institute, Northern Technical University, Mosul, Iraq

\section{Introduction}

Melioidosis is a serious disease with a wide range of manifestations, depending on the route of infection. The causative agent, Burkholderia pseudomallei, is a Gram-negative bacillus that is endemic in the tropics [1,2]. The bacterium is normally resident in soil and acts as an opportunistic pathogen. There is little evidence of person:person transmission and infection occurs after exposure to contaminated soil/aerosols: increased risk factors for contracting the disease include diabetes and excessive alcohol consumption [3]. Most notified cases of melioidosis are in South East Asia and northern Australia, but underreporting is likely exacerbated by its similarity to other infectious conditions (notably tuberculosis). Despite recent estimates that it causes more fatalities (89,000 per annum) than Dengue fever [2], melioidosis has thus been relatively neglected and its prevalence underestimated. Infection can remain dormant for long 
periods [4]; there is no effective vaccine and the bacterium is resistant to many antibiotics.

Burkholderia pseudomallei is a facultative intracellular pathogen and can invade a wide range of tissues, giving rise to diverse clinical manifestations including pneumonia, septicaemia, abscesses, acute pyelonephritis, osteomyelitis, and encephalitis [2]. After cell invasion, the bacteria are able to escape from the endocytic compartment into the cytosol where they replicate and acquire mobility by inducing actin polymerisation [5, 6]. Unusually amongst bacteria, B. pseudomallei are able to induce fusion of the infected cells with non-infected cells to form multinucleated giant cells (MNGC) [7]. Such MNGC or syncytia are observed in the tissue of patients with melioidosis [8] and the bacterium is also able to induce MNGC formation in vitro in a variety of mammalian cell lines $[9,10]$. MNGC formation can similarly be induced by the closely related but relatively non-pathogenic species, Burkholderia thailandensis [10]. In melioidosis, the capacity to form MNGC is thought to be associated with pathogenicity and may facilitate cell:cell spread, evasion of the immune response and might also protect against antibiotics [5-7]. The type VI secretion system 5 (T6SS-5), which is associated with virulence in animal models of infection, is required for cell:cell fusion in both $B$. pseudomallei and B. thailandensis [11, 12]. It is very likely, however, that host cell factors are also involved in MNGC formation and it has been shown that antibodies to certain host cell surface proteins inhibit cell fusion induced by $B$. pseudomallei in human U937 macrophages [13].

The tetraspanins are a family of evolutionarily conserved membrane proteins with 33 members in humans and a similar number in mice [14]. They are involved in many basic cell functions and act primarily by associating with and organising the other membrane proteins to form functional microdomains known as TEM (tetraspanin-enriched microdomains) [15]. Tetraspanins have been implicated in the control of cell:cell fusion, perhaps most notably tetraspanin CD9 in sperm:egg fusion, with female mice showing greatly reduced fertility owing to the inability of the oocytes to fuse [16]. Loss of the tetraspanin CD81 exacerbates this phenotype [17]. Tetraspanins have also been shown to be involved in the control of muscle cell fusion [18], osteoclast formation [19], mononuclear phagocyte MNGC formation [20-22], and virus-induced syncytium formation [23-25].

Given their involvement in other types of infectious and non-infectious cell:cell fusion, it was, therefore, of interest to determine if tetraspanins might play a role in fusion induced by Burkholderia species. B. pseudomallei is classed as Tier 1 bioweapon [2], but the components of the T6SS-5 machinery involved in promoting MNGC formation are very similar in the rarely pathogenic but closely related species $B$. thailandensis [26]. We, therefore, investigated the role of tetraspanins CD9, CD63, and CD81 in MNGC formation induced by this bacterium in mouse macrophage cell lines. Two $B$. thailandensis isolates were used: E264, an environmental isolate [27], and CDC272, a clinical isolate [28]. The effects of specific anti-tetraspanin antibodies and recombinant proteins representing the large extracellular region (EC2) on B. thailandensisinduced MNGC formation were examined. In addition, we investigated MNGC formation in infected mouse macrophages where the tetraspanin CD9 had been ablated or was overexpressed. We also conducted preliminary investigations on other cell surface molecules that have been implicated in mononuclear phagocyte MNGC formation, some of which are known to associate with tetraspanins. Overall, our findings demonstrate that tetraspanins act to regulate MNGC formation induced by $B$. thailandensis in mouse macrophages, with CD9 in particular acting as a negative regulator of this process. Given the similarity between MNGC formation induced by $B$. thailandensis and. B. pseudomallei, our findings may have implications for the disease mechanisms involved in melioidosis.

\section{Materials and methods}

\section{Mammalian cell lines}

The J774.2 mouse macrophage cell line was originally obtained from Prof H. Harris and Dr R. Sutherland (Sir William Dunn School of Pathology Oxford) and cultured in DMEM $+2 \mathrm{mM}$ glutamine $+4.5 \mathrm{~g} / \mathrm{l}$ glucose (Gibco) with 10\% FCS (Biowest). The RAW 264.7 mouse macrophage cell line was from the American Type Culture Collection (ATCC) and cultured in DMEM with GlutaMAX (Gibco) with 10\% FCS. J774.2 stably overexpressing mouse CD9GFP and GFP were generated by transfection with pCMV6AV-mCD9-GFP or pCMV-AV-GFP (Origene) using electroporation followed by selection in G418-containing medium and further selection by fluorescence activated cell sorting (FACS Aria, Becton-Dickinson). CD9WT and CD9 null macrophage cell lines were derived from CD9 knockout and the corresponding wild-type C57BL/6 mice [29] by transformation of peritoneal mouse macrophages using J2-transforming retrovirus [30]. These cell lines were kindly provided by Dr. Gabriela Dveksler, Dept. Pathology, Uniformed Services University of Health Sciences, Bethesda, $\mathrm{MD}$, US and cultured in DMEM + $2 \mathrm{mM}$ Glutamine $+4.5 \mathrm{~g} / \mathrm{l}$ glucose $+10 \%$ FCS. CD82WT and CD82 null mouse macrophage cell lines were derived from CD82 knock-out and the corresponding wild-type C57BL/6 mice [31]. These cells were kindly provided by Professor Jatin Vyas, Division of Infectious Disease, Massachusetts General Hospital, Boston, US. All cells were maintained at $37^{\circ} \mathrm{C}$ under $5 \% \mathrm{CO}_{2}$. 


\section{Burkholderia thailandensis strains}

E264, an environmental isolate, sequenced strain [27, 32] and CDC2721121, a clinical isolate from Louisiana, abbreviated as CDC272 [28] were kind gifts from the laboratory of Professor Richard Titball, Dept. Biosciences, University of Exeter, UK.

\section{Antibodies}

Monoclonal antibodies $(\mathrm{mAb})$ against mouse tetraspanins were CD9 (Cat. No. MCA2749, clone MF1 Bio-Rad), CD63 (Cat. No. 143902, clone NVG-2, Biolegend), CD81 (Cat. No. MCA1846, clone Eat2 Bio-Rad), and matching isotype controls rat IgG2b (Bio-Rad), rat IgG2ak (Biolegend) and hamster IgG1 (Bio-Rad), respectively. MAb to other cell surface molecules were CD36 (Cat. No. 102602, clone HM36), CD44 (Cat. No. 103002, clone IM7) CD47 (Cat. No. 127502, clone miap301) CD98 (Cat. No. 128202, clone RL388), CD172a (Cat. No. 144002, clone P84) (all from Biolegend), and DC-STAMP (Cat. No. MABF39, clone 1A2 Millipore). Isotype controls were Armenian hamster IgG, rat

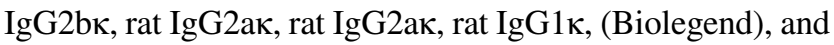
$\mathrm{IgG} 2 \mathrm{a}$ (Millipore), respectively. The secondary antibodies used for flow cytometry were anti-rat IgG-FITC (Cat. No. F-9387, Sigma) and anti-hamster IgG-FITC (Cat. No. MCA2357, Bio-Rad). All antibodies were used at saturating binding concentrations.

\section{Recombinant GST-EC2 proteins}

The glutathione S-transferase (GST) fusion system was used to produce GST-tagged tetraspanin EC2 proteins as described previously $[22,33]$.

\section{Invasion assay}

The invasion and intracellular survival of $B$. thailandensis was assessed using a modified kanamycin protection assay [10]. Cells were seeded at $2 \times 10^{5}$ cells $/ \mathrm{ml}$ in 24 -well plates and cultured overnight. An overnight culture of bacteria was washed twice with PBS with centrifugation and the pellet suspended to $\mathrm{OD} \sim 0.4$. Cells were infected at a multiplicity of infection (MOI) of 3:1 (determined after optimisation) and were incubated at $37{ }^{\circ} \mathrm{C} 5 \% \mathrm{CO}_{2}$ for $2 \mathrm{~h}$. Cells were then washed with PBS and incubated with media containing $500 \mu \mathrm{g} / \mathrm{ml} \mathrm{kanamycin} \mathrm{and} 500 \mu \mathrm{g} / \mathrm{ml}$ amikacin for an additional $2 \mathrm{~h}$ to eliminate extracellular bacteria. As a negative control, cells were treated with cytochalasin D (Sigma) for $1 \mathrm{~h}$ prior to infection to prevent bacterial uptake; also the supernatant from the cells was examined for viable bacteria. Cells were then washed with PBS and lysed in $0.01 \%$ Triton $\mathrm{X}-100$ in PBS for $5 \mathrm{~min}$. The lysis mixture was diluted and a selection of dilutions were plated on LB agar plates and then incubated at $37^{\circ} \mathrm{C}$. The number of intracellular bacteria was quantified after $28 \mathrm{~h}$ of incubation.

\section{Multinucleated giant cell formation assay}

Cells were infected and extracellular bacteria later eliminated as described above. After an appropriate time postinfection, cells were washed with PBS and fixed using acid/ ethanol [5\% acetic acid (v/v), $5 \% \mathrm{dH}_{2} \mathrm{O}$, and $90 \%$ ethanol $(\mathrm{v} / \mathrm{v})$ ] for $30 \mathrm{~min}$ at RT. Cells were washed with PBS and stained with Giemsa solution (Sigma) $(0.1 \%$ solution $w / v)$ for $30 \mathrm{~min}$ at RT, then washed with $\mathrm{dH}_{2} \mathrm{O}$, and allowed to dry. Images were captured with a Nikon light microscope using the $40 \mathrm{X}$ objective.

\section{Evaluation of multinucleated giant cell formation}

Images were analysed using Image $\mathbf{J}$ software. For each experimental condition, images of 10 random fields captured at $400 \times$ magnification were analysed and cells with $>3$ nuclei were considered to be MNGCs. Data from all 10 fields were combined, and then, the percentage of MNGCs and the average MNGC size were calculated as described previously [21].

\section{Effect of $m A b$ and GST-EC2 proteins on $B$. thailandensis-induced MNGC formation}

Macrophages were seeded at $2 \times 10^{5}$ in 96 -well plates $\left(100 \mu \mathrm{l} /\right.$ well) and incubated overnight at $37{ }^{\circ} \mathrm{C} 5 \% \mathrm{CO}_{2}$. The cells were treated with $\mathrm{mAb}$ at $10 \mu \mathrm{g} / \mathrm{ml}$ (saturating binding concentration as determined by titration) or EC2 proteins at $500 \mathrm{nM}$ for $1 \mathrm{~h}$ before the infection. The MNGC assays were then carried out as described above.

\section{Flow cytometric analysis of antigen expression}

The expression of tetraspanins and other cell surface molecules was assessed by flow cytometry. Cells were harvested, pelleted, and resuspended with wash buffer (HBSS (Lonza) containing $0.2 \%$ BSA (Sigma) and $0.1 \%$ sodium azide), and then transferred to flow cytometry tubes (Elkay) at $10^{6} \%$ tube. After centrifugation, the cell pellet was incubated with appropriate primary antibody or isotype control at $10 \mu \mathrm{g} / \mathrm{ml}$ for $1 \mathrm{~h}$ on ice. After washing twice, the cells were incubated with appropriate FITC-labelled secondary antibody (anti-rat IgG-FITC (AbCam) or anti-hamster IgG-FITC (Abcam)) for $1 \mathrm{~h}$ on ice. Cells were then analysed using an FACS LSR II (Becton-Dickinson). Data were analysed with FlowJo, LLC software. In the case of infected cells, cells were fixed with $1 \%$ paraformaldehyde for $30 \mathrm{~min}$ prior to analysis. Intracellular expression of CD63 was assessed following cell 
permeabilization using Fix and Perm (Caltag) according to the manufacturer's instructions.

\section{Statistical analysis}

Unless otherwise stated, all data presented represent at least three independent experiments. Graphs were drawn and statistical analyses performed using Graphpad Prism version 8.3.1 (Graphpad Software, San Diego USA). Details of the statistical analyses used are given in the figure legends.

\section{Results}

\section{Induction of MNGC formation by B. thailandensis in macrophage cell lines}

It has been reported that $B$. pseudomallei can induce MNGC formation in phorbol myristate acetate (PMA) differentiated human U937 cells [13]. Our initial investigations showed that whilst undifferentiated or PMA-differentiated U937 cells and THP1 cells were infected by E264 and CDC72 strains of $B$. thailandensis, no MNGC formation was observed in these human mononuclear phagocyte cell lines. This may reflect differences in virulence between the bacterial species. We, therefore, turned our attention to mouse macrophage cell lines, where MNGC formation by $B$. thailandensis $\mathrm{E} 264$ and $B$. thailandensis $\mathrm{CDC} 272$ has previously been reported [10]. Both strains of $B$. thailandensis induced MNGC formation in mouse macrophage cell lines J774.2 and RAW264.7, with fusion evident at $6 \mathrm{~h}$ post-infection and reaching a maximum at $18-24 \mathrm{~h}$ (Fig. 1). After this time, the infected cells appeared to undergo necrosis. The rates and levels of MNGC formation that we observed with these bacterial strains and cell lines are similar to those previously reported [10], although we saw slightly higher levels of MNGC formation with the environmental E264 strain. After the initial optimisation, infection of the macrophages was thereafter carried out at an MOI of 3:1 for $2 \mathrm{~h}$, followed by treatment with antibiotics to kill extracellular bacteria as described in Materials and Methods, and MNGC formation was assessed after $16 \mathrm{~h}$.

\section{Effect of anti-tetraspanin antibodies on $B$. thailandensis-induced MNGC formation}

Initially, the expression of tetraspanins by J774.2 and RAW264.7 mouse macrophages was investigated using commercially available mAb to mouse CD9, CD63 and CD81. These tetraspanins were of interest, since the previous work from our group and others [20-22] have indicated that they are involved in mononuclear phagocyte MNGC formation in response to non-infectious stimuli. The relative levels of cell surface expression of these tetraspanins on the two cell lines, as determined by flow cytometry, are shown in Fig. 2 . Both cell lines express relatively high levels of CD9 and CD81, with lower expression of CD63 on RAW264.7 cells. CD63 expression is comparable to isotype control values on J774.2 cells, although some of this antigen is present intracellularly; this is not unexpected as CD63 is known to have predominantly intracellular location [34].

The effects of the mAb in both macrophage lines was investigated by treating the cells with saturating binding concentrations of test antibody or appropriate matched isotype controls for $1 \mathrm{~h}$ prior to infection with either the $B$. thailandensis $\mathrm{E} 264$ or $\mathrm{CDC} 272$ strains, followed by the assessment of MNGC formation as described above. The results showing the effects of the anti-tetraspanin $\mathrm{mAb}$ relative to the isotype-matched controls are depicted in Fig. 3A, B. mAb to CD9 and CD81 enhanced B. thailandensis CDC272-induced MNGC formation in both RAW264.7 and J774.2 mouse macrophages, with a significant increase in the percentage of fused cells for both cell lines and an increase in MNGC size for anti-CD81 antibodies. The anti-CD9 and antiCD81 mAb also significantly enhanced MNGC formation in RAW264.7 cells in response to E264 cells, and an effect was also observed here with anti-CD63, with a significant increase in the percentage of MNGC compared to the corresponding isotype control (Fig. 3B). A significant increase in MNGC size was also observed with anti-CD9 and anti-CD81 in J774.2 cells infected with E264, although the increase in the percentage of fused cells did not reach significance here. The lack of an effect of anti-CD63 antibodies on infectioninduced MNGC formation in J774.2 macrophages is perhaps unsurprising given the very low levels of this antigen on the surface of these cells. The antibodies did not affect invasion by either strain of $B$. thailandensis of mouse macrophages with (Fig. 3C), indicating that their effects are specifically related to cell fusion rather than the initial adhesion and infection. It should be noted that treatment of the macrophages with the anti-tetraspanin antibodies alone (i.e., in the absence of infection) had no effect on the cells.

\section{Effect of antibodies to non-tetraspanin fusion-associated proteins on $B$. thailandensis-induced MNGC formation}

A number of non-tetraspanin proteins have been implicated in MNGC formation and it was of interest to examine the effects of $\mathrm{mAb}$ to these proteins on cell:cell fusion induced by B. thailandensis (Fig. 4). Some of these proteins are known to interact with tetraspanins in the context of TEM. In addition, antibodies to integrin-associated protein CD47 and fusion regulatory protein CD98 have previously been shown to inhibit $B$. pseudomallei-induced fusion in human U937 cells [13]. Here, we found that in 
Fig. 1 MNGC formation induced by $B$. thailandensis strains E264 and CDC272 in J774.2 and RAW264.7 mouse macrophages. A Time-course of MNGC formation in J774.2 (upper panel) and RAW264.7 (lower panel) mouse macrophages induced by $B$. thailandensis strains E264 and CDC272. Cells were stained with Giemsa, and the number of MNGC (cells with $>3$ nuclei) was determined visually per field of view and expressed as a percentage of the total number of macrophages. The average size of MNGC was also calculated by determining the number of nuclei per MNGC per field of view. The error bars represent the standard error of the mean from three independent experiments, each comprising ten fields of view. B Representative images of Giemsa stained uninfected RAW264.7 cells (a); cells infected with $B$. thailandensis CDC272 (b); or E264 (c) for $16 \mathrm{~h}$; and with CDC272 for $24 \mathrm{~h}$ showing cell necrosis (d). Images were captured using by light microscopy using the $\times 40$ objective, scale bar $20 \mu \mathrm{m}$
A
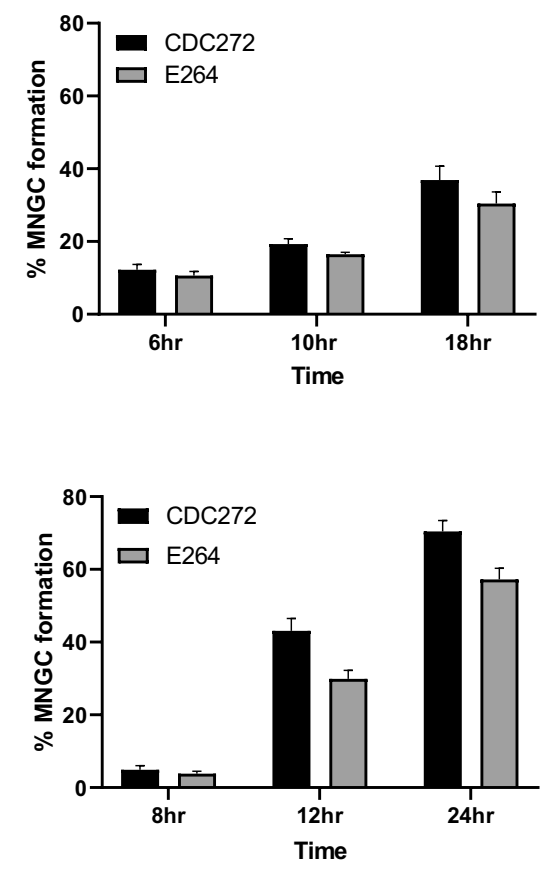

$\mathbf{J} 774.2$

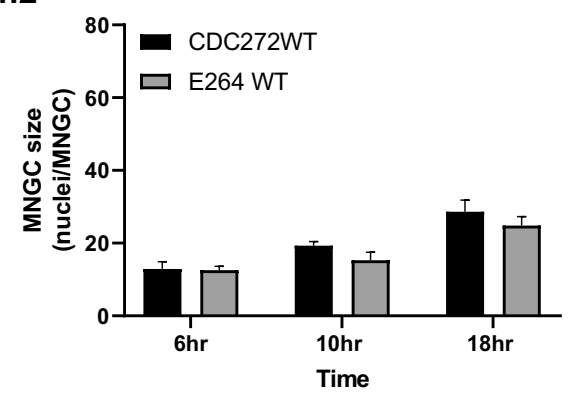

RAW264.7

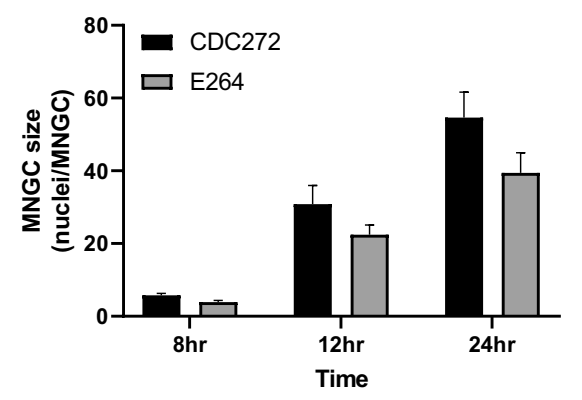

\section{B}

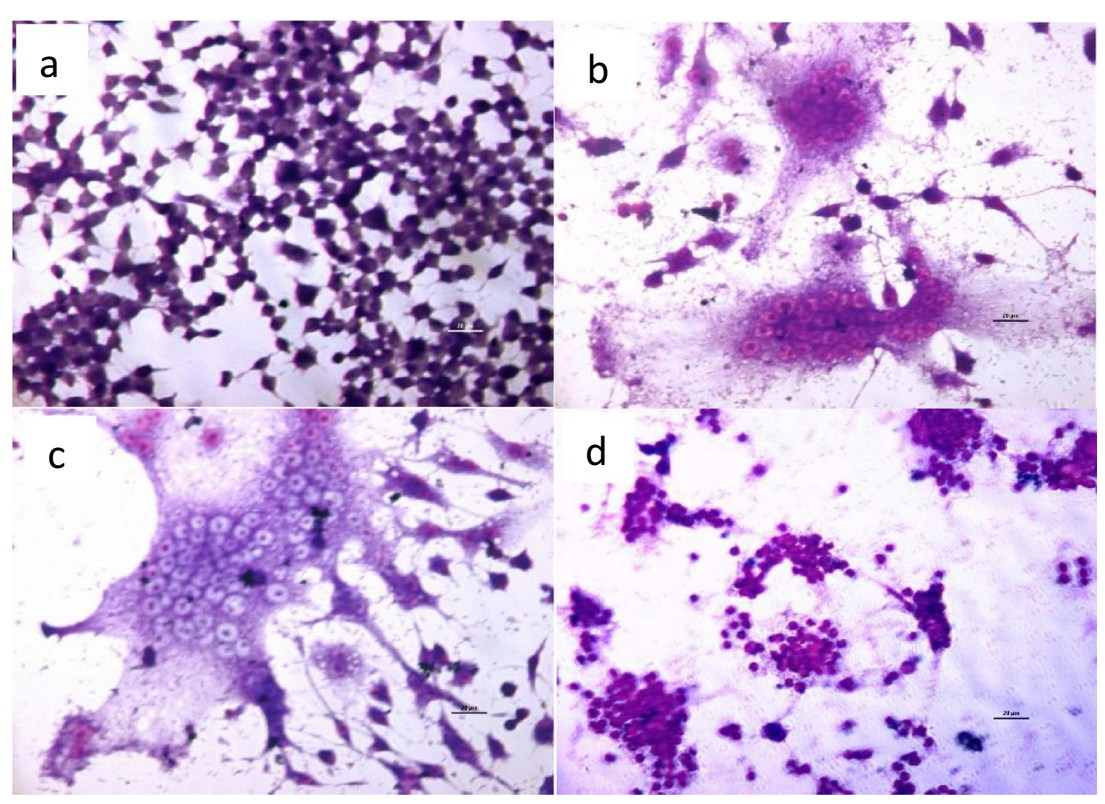

J774. 2 cells, antibodies to CD47 and DC-STAMP (a protein deemed essential for osteoclast fusion [35]) significantly inhibited MNGC formation induced by both strains of B. thailandensis (Fig. 4A) and an antibody to CD98 also significantly inhibited CDC272-induced MNGC formation. By contrast, an antibody to CD44 enhanced MNGC formation to a significant extent in cells infected with the E264 strain.

We also used flow cytometry to investigate the early changes in the cell surface expression of these proteins and tetraspanins CD9 and CD81 following infection (Fig. 4B). A significant decrease in expression of CD9 was observed $3 \mathrm{~h}$ after infection of $\mathbf{J} 774.2$ macrophages with B. thailandensis CDC272 compared with uninfected cells. There was a suggestion that CD44 also decreased on infected cells, but this did not reach significance. Attempts were made to investigate changes in antigen expression at later time points after infection; however, we observed that once the macrophages start to fuse, they become extremely adherent and we were unable to detach the cells for flow cytometry 


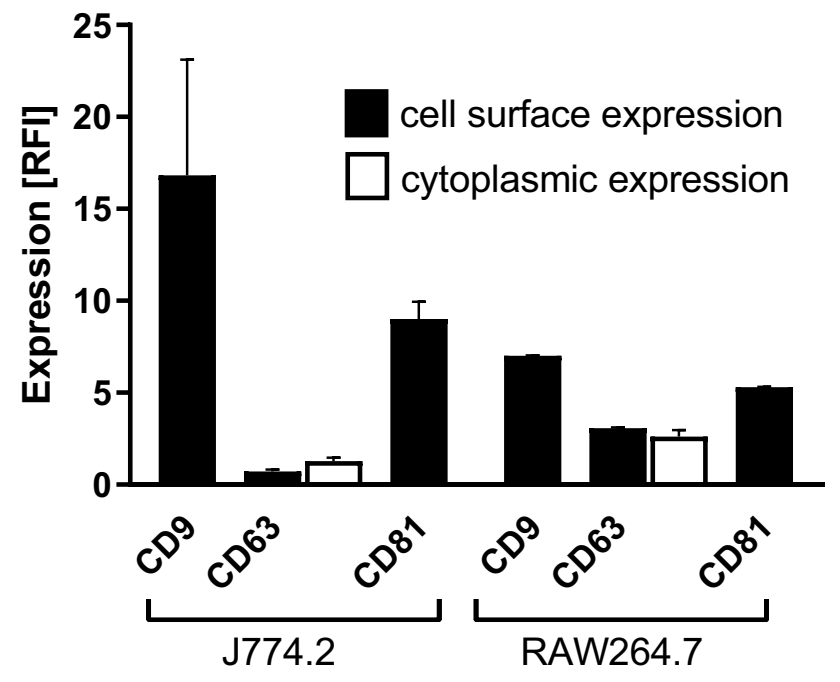

Fig. 2 Expression of CD9, CD63, and CD81 tetraspanins by J774.2 and RAW264.7 mouse macrophages. The relative levels of expression were measured by flow cytometry and are expressed as Relative Fluorescence Intensity (RFI), i.e., MFI of anti-tetraspanin antibody/ MFI isotype control. The data represent at least two experiments performed in duplicate. The data are for live (unfixed) cells except for data shown for CD63 (white fill) which depicts cytoplasmic staining determined using fixed and permeabilized cells

without damaging them. This is consistent with the observations that $B$. thailandensis-infected macrophages adopt an osteoclast-like phenotype [36].

\section{Effect of recombinant EC2 proteins on $B$. thailandensis-induced MNGC formation}

Since the effect of mAb on E264-induced MNGC formation in J774.2 cells was less marked than that with the CDC272 strain of $B$. thailandensis, we further explored the involvement of tetraspanins in this macrophage cell line using recombinant proteins corresponding to the large extracellular domains (EC2s) of tetraspanins fused to GST. The GSTEC2 proteins are known to fold correctly and have shown biological activity in a wide range of assays [23, 33, 37, 38], including mononuclear phagocyte fusion [20-22]. Pre-treatment of J774.2 macrophages with GST-EC2s corresponding to $\mathrm{CD} 9, \mathrm{CD} 63$, and CD81 significantly suppressed MNGC formation on infection with both E264 and CDC272 strain of B. thailandensis (Fig. 5A). Pre-treatment of J774.2 cells with the recombinant proteins did not inhibit bacterial invasion; GST-EC2CD9 gave a slight but significant increase in bacterial invasion (Fig. 5B), again suggesting that the inhibitory effects are specific to the cell:cell fusion process. These findings thus confirm roles for CD9 and CD81 in cell:cell fusion induced by both strains of B. thailandensis in J774.2 macrophages and also suggest a role for CD63, despite the low surface expression of this tetraspanin on these cells.
Interestingly, CD63 has previously been implicated in mononuclear phagocyte fusion induced by Con A [21].

\section{CD9 KO enhances $B$. thailandensis-induced MNGC formation, whereas CD9 overexpression suppresses B. thailandensis-induced MNGC formation}

A mouse macrophage cell line derived from a CD9 null mouse and a corresponding WT control [30] were examined for their susceptibility to MNGC formation in response to infection with both strains of B. thailandensis (Fig. 6A). A significant increase in fusion index was apparent in the CD9 null macrophages with both CDC272 and E264 strains and an increase in average MNGC size that was significant for CDC272. A more detailed time-course showed that the CD9 null macrophages underwent cell:cell fusion at an earlier time point than the WT macrophages, with accelerated MNGC formation from 8 to $20 \mathrm{~h}$ (Fig. 6B). The CD9 null macrophages did not appear to be significantly more susceptible to infection with B. thailandensis (Fig. 6C), indicating that the ablation of CD9 affected cell fusion directly.

Since CD9 is known to interact with the other cell surface proteins in the context of TEM and may regulate their expression, a comparison of the cell surface expression of tetraspanins CD63 and CD81, along with that of markers that have been commonly implicated in mononuclear phagocyte fusion [39], was made using flow cytometry (Fig. 6D). Of the fusion-associated markers, only CD36 (also known as scavenger receptor and implicated in some types of macrophage fusion [40]) showed a significant difference in expression, with approximately 1.5 -fold lower expression on the CD9 null macrophages. Interestingly, the cell surface expression of the tetraspanin CD81 was also significantly reduced on the CD9 null macrophages. It should be noted that a comparison of the gene expression profiles of the CD9 null and WT macrophage cell lines by microarray analysis showed no obviously relevant changes in gene expression, except for the expected decrease in CD9 mRNA in the CD9 null cells (data not shown).

Studies were also carried out on a mouse macrophage cell line derived from a CD82 null mouse and the corresponding WT control (Fig. 7). Interestingly, the CD82 null macrophages were significantly more prone to forming MNGC on infection with both strains of B. thailandensis (Fig. 7A). Again, there was little difference in susceptibility to infection between the cell lines, with CD82 null cells if anything showing reduced infection (Fig. 7B).

To further investigate the role of CD9 in bacterially induced MNGC formation and infection, assays were performed using J774.2 macrophages that had been stably transfected to overexpress mouse CD9-GFP or GFP alone. The CD9-GFP-transfected macrophages showed a significant reduction in MNGC formation (approximately 40\%) for both 

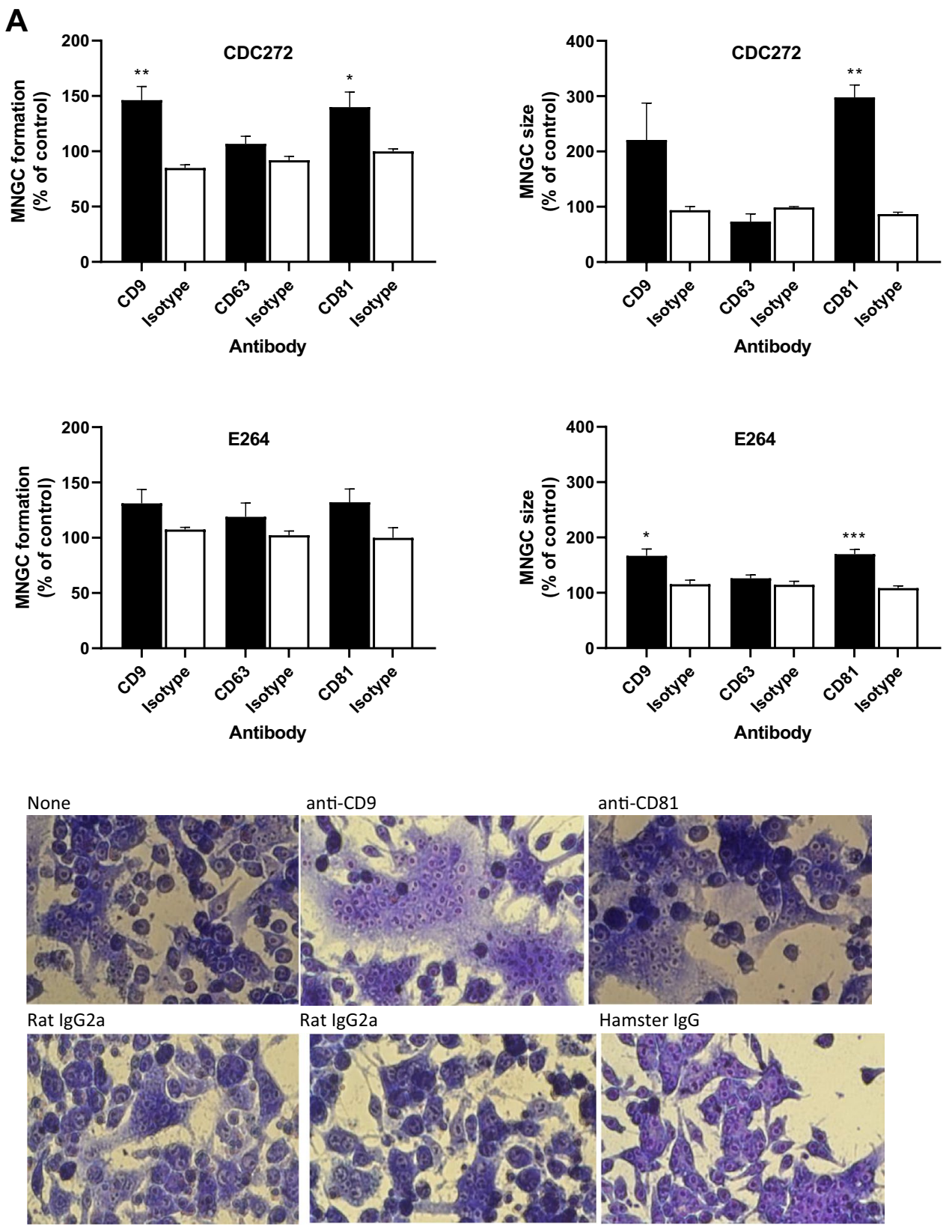

Fig. 3 Effect of $\mathrm{mAb}$ to $\mathrm{CD} 9, \mathrm{CD} 63$, and CD81 on MNGC formation induced by B. thailandensis strains CDC272 and E264 in J774.2 (A) and RAW264.7 macrophages (B). Macrophages were pre-treated with test $\mathrm{mAb}$ or isotype-matched controls for $1 \mathrm{~h}$ prior to infection with bacteria and MNGC formation was assessed as described previously. The data are presented as a percentages of the MNGC formed (\% of MNGC) (left panel) or average MNGC size (right panel) compared to the untreated (no antibody) control. The results are representative of at least three independent experiments, with error bars showing standard error of the mean. Statistical analysis was carried out between the test mAb (black fill) and the matched isotype control (white fill) using Welch's $t$ test. $* p<0.05$, $* * p<0.001$,
$* * * p<0.0001$. Pairs with no asterisk showed no significant difference. Representative images of untreated, anti-CD9, anti-CD81, and isotype control-treated J774.2 cells infected with CDC272 and fixed and stained after $16 \mathrm{~h}$ are shown in the lower panel of $(\mathbf{B})$. $\mathbf{C}$ Effect of $\mathrm{mAb}$ to the tetraspanins (black fill) and corresponding isotype control (white fill) on B. thailandensis infection. J774.2 cells were pre-incubated with antibodies as described above prior to infection $(2 \mathrm{~h})$ and after kanamycin protection to kill extracellular bacteria $(2 \mathrm{~h})$, macrophages were lysed, and the number of surviving intracellular bacteria determined (cfu/10,000 host cells). Data were analysed as for (A, B), but no significant differences were found between anti-tetraspanin $\mathrm{mAb}$ and the isotype control-treated samples 

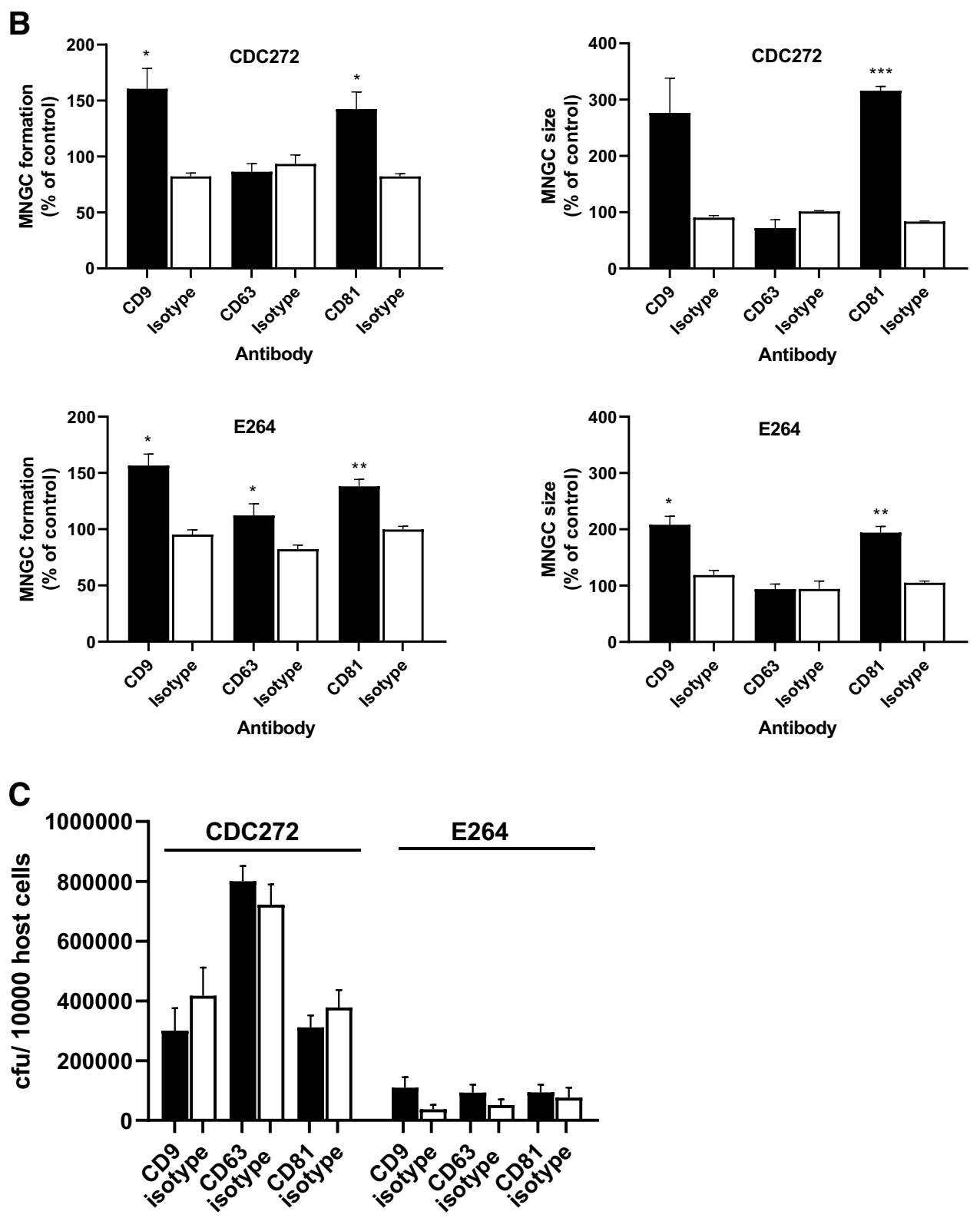

Fig. 3 (continued)

CDC272 and E264 strains of B. thailandensis compared to WT or GFP-transfected J774.2 cells (Fig. 8A), although neither of the transfected cells differed in susceptibility to infection compared with wild-type J774.2 macrophages (Fig. 8B).

\section{Discussion}

The disease melioidosis is caused by the Gram-negative environmental bacterium Burkholderia pseudomallei, with the highest incidences recorded in South East Asia and northern Australia. Underreporting of the condition is very likely, however, and recent estimates suggest that melioidosis may be globally responsible for 89,000 deaths per year [2]. The closely related species $B$. thailandensis has been reported to cause disease in man in only a handful of cases, but shares many of the features of $B$. pseudomallei. $B$. thailandensis is commonly used as a model for infection and it causes disease in mice [27]. Of relevance to the present study, B. thailandensis shares with $B$. pseudomallei the ability to induce MNGC formation and the bacterial factors involved in cell:cell fusion, notably the T6SS-5 secretion system, are very similar between the species [12]. This feature, which is associated with virulence in B. pseudomallei, 
Fig. 4 Effect of non-tetraspanin antibodies on B. thailandensisinduced MNGC formation in J774.2 macrophages. A Macrophages were pre-treated with test $\mathrm{mAb}$ or isotype-matched control for $1 \mathrm{~h}$ prior to infection with bacteria and MNGC formation was assessed as described previously. The data are presented as percentages of MNGC formed (\% MNGC) (left panel) or average MNGC size (right panel) compared to the untreated (no antibody) control. The results are representative of at least three independent experiments, with error bars showing standard error of the mean. Statistical analysis was carried out between the test $\mathrm{mAb}$ (black fill) and the matched isotype control (white fill) using Welch's $t$ test. * $p<0.05$, $* * p<0.001$. Pairs with no asterisk showed no significant difference. B Changes in cell surface expression of antigens $3 \mathrm{~h}$ after infection of $\mathbf{J} 774.2$ cells with $B$. thailandensis CDC272 was assessed by flow cytometry relative to uninfected controls at the same point. The data represent RFI values from at least four independent experiments performed in duplicate. Statistical analysis was carried out between the uninfected (black fill) and infected (white fill) cells using Welch's $t$ test. ${ }^{*} p<0.05$. Pairs with no asterisk showed no significant difference
A
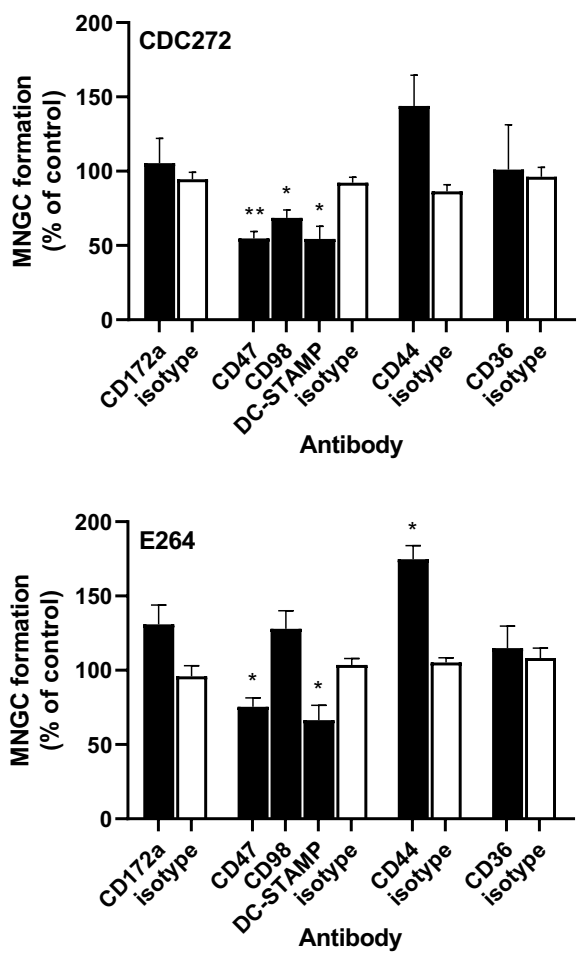
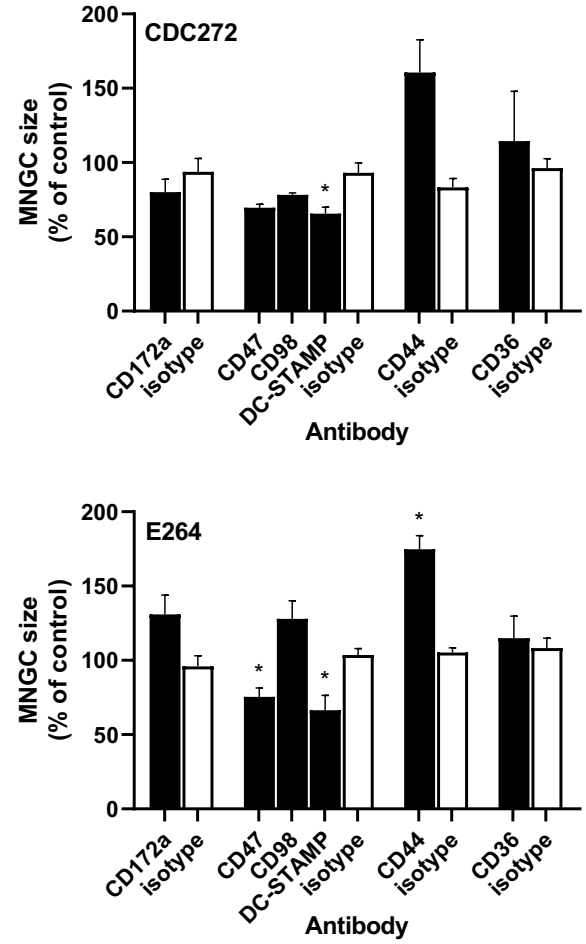

B

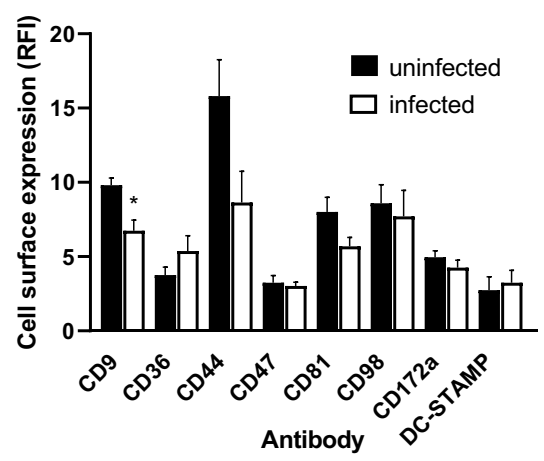

is likely to be regulated by host cell factors including cell surface proteins.

Members of the tetraspanin superfamily of membrane proteins have been implicated in naturally occurring cell:cell fusion (e.g., sperm:egg fusion, myoblast, and osteoclast formation) [41], as well as MNGC formed by mononuclear phagocytes in response to inflammation [20-22] and virusinduced syncytial formation [24, 25]. We, therefore, investigated the role of tetraspanins reported to regulate cell:cell fusion in these systems in B. thailandensis-induced MNGC formation in mouse macrophages.

MNGC formation was successfully induced in J774.2 and RAW264.7 macrophages following infection with CDC272 (a clinical isolate) and E264 (an environmental isolate) strains of $B$. thailandensis, in line with a previous study

[10]. These macrophage cell lines expressed relatively high levels of the tetraspanins CD9 and CD81, which are reported to regulate non-infectious mononuclear phagocyte fusion. A significant enhancement of MNGC formation following treatment of J774.2 and RAW264.7 cells with anti-CD9 and anti-CD81 $\mathrm{mAb}$ prior to infection with $B$. thailandensis was observed (Fig. 3). This pattern, which did not relate to an effect on infection per se, was very similar to the effects of antibodies seen on the other forms of MNGC formation in mononuclear phagocytes [20-22]. Conversely, recombinant proteins corresponding to the large extracellular domain (EC2) of CD9, CD63, and CD81 significantly inhibited $B$. thailandensis-induced MNGC formation in J774.2 cells, suggesting overall that these tetraspanins act as negative regulators of fusion. To explore this further, we investigated 

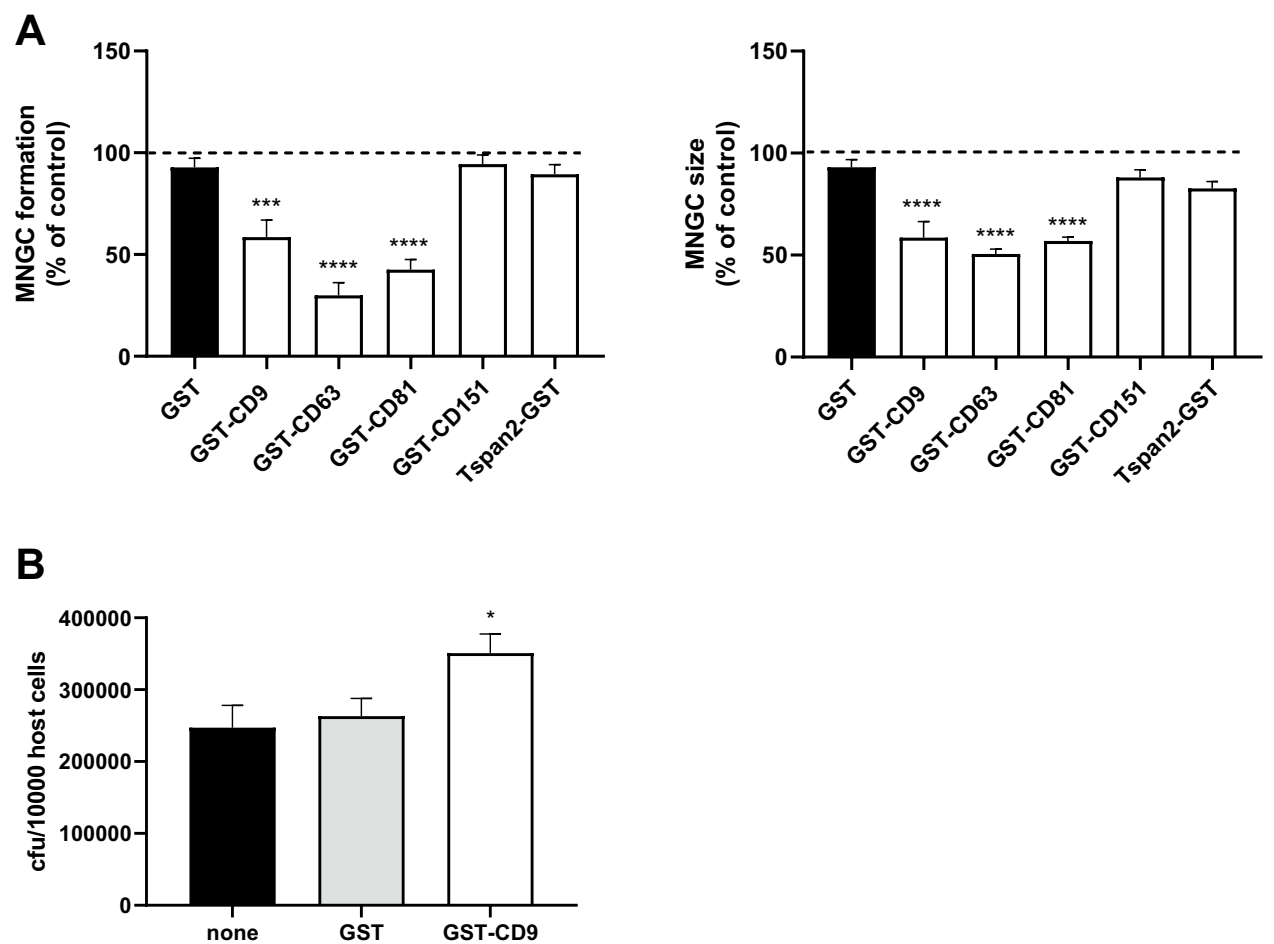

Fig. 5 Effect of recombinant GST-EC2 on MNGC formation induced by $B$. thailandensis strains $\mathrm{CDC} 272$ and E264 in J774.2 macrophages. A Macrophages were pre-treated with GST or GST-EC2 proteins for $1 \mathrm{~h}$ prior to infection with bacteria and MNGC formation was assessed as described previously. The data are presented as the percentages of MNGC formed (\% MNGC) (left panel) or average MNGC size (right panel) compared to the untreated (no recombinant protein added) control. The results are representative of at least three independent experiments, with error bars showing standard error of

MNGC formation following $B$. thailandensis infection of macrophages derived from CD9 null and wild-type mice. A significant enhancement of MNGC formation was observed with CD9 null infected macrophages, although these cells did not appear to show any increase in overall infection. This is again strongly indicative of a negative regulatory role for tetraspanin CD9 in bacterial-induced fusion. Consistent with this, J774.2 macrophages overexpressing CD9 showed significantly reduced MNGC formation on infection. Interestingly, the initial investigations showed that macrophages from CD82 null mice were also more prone to forming MNGC on infection with $B$. thailandensis. To our knowledge, there are currently no mAb specific to mouse CD82, but it would be informative to investigate this further should such reagents become available.

CD9, in common with other tetraspanins, is known to interact with the other cell surface proteins in TEM and to regulate their localisation/activity. To try to explore this further, we examined the expression of other molecules implicated cell:cell fusion in our system. CD36, a member of the class B scavenger receptor family, has been the mean. Significance of difference from GST control was established by one-way ANOVA with Dunnett's post-test. $* * * p<0.001$; $* * * * p<0.0001$. B shows the effect of GST proteins on B. thailandensis infection. J774.2 cells were pre-incubated with or without the recombinant proteins prior to infection $(2 \mathrm{~h})$ and after kanamycin protection to remove extracellular bacteria $(2 \mathrm{~h})$, macrophages were lysed and the number of surviving intracellular bacteria determined (cfu/10,000 host cells). Significance of difference from GST control was established as described for (A)

implicated in cytokine-induced mononuclear phagocyte fusion [40] and myoblast fusion [42]. A slight reduction in the surface expression of this protein was evident on CD9 null macrophages, but antibodies to CD36 had no effect on B. thailandensis-induced MNGC formation and no change in expression was observed on macrophage infection with $B$ thailandensis. Interestingly, CD36 has been reported to associate closely with CD9 on the surface of mononuclear phagocytes [43]. CD44 is a widely expressed cell surface protein with roles in cell adhesion and cell:cell interactions. CD44 has also been implicated in cell fusion processes, with ligands and $\mathrm{mAb}$ reported to inhibit osteoclast formation [44, 45], whilst bone marrow cells from CD44 KO mice showed enhanced osteoclast formation in vitro [46]. We observed that antibodies to CD44 gave some enhancement of $B$. thailandensis-induced MNGC formation; this mirrors the effects of $\mathrm{mAb}$ to $\mathrm{CD} 9$ and $\mathrm{CD} 81$, suggesting that CD44 may also act as a negative regulator of fusion here. Interestingly, CD44 is known to associate with CD9 in TEM [47]. CD47 is an integrin-associated protein that is reported to be important in macrophage fusion [48]. 


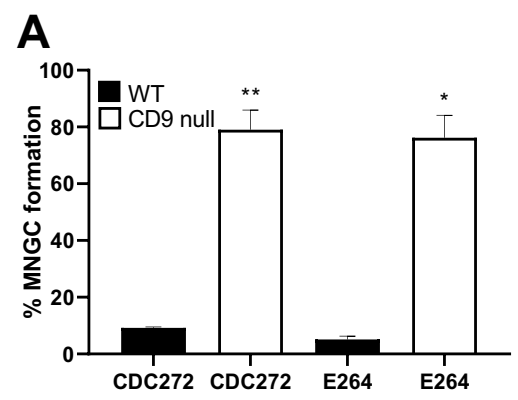

B

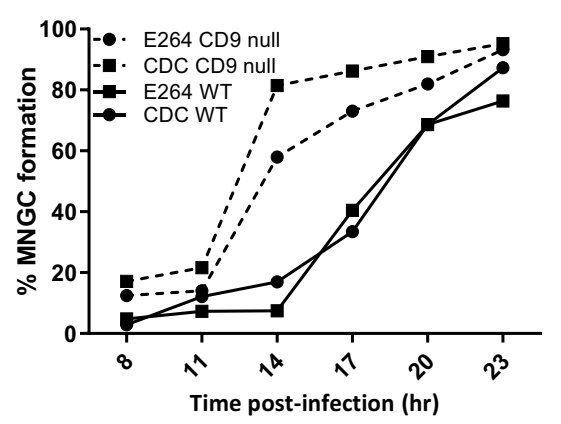

C

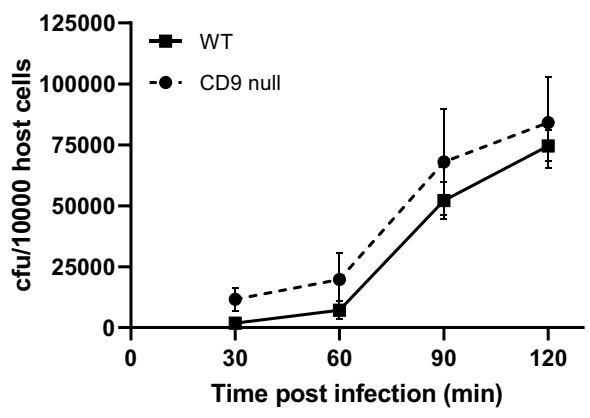

Fig. 6 Effect of CD9 knock-out on MNGC formation induced by $B$. thailandensis strains CDC272 and E264 in mouse macrophages. A Following infection of macrophages derived from WT (black fill) or CD9 null mice (white fill) with both strains of bacteria, MNGC formation was assessed after $14 \mathrm{~h}$ as described previously. The results are representative of three independent experiments, with error bars showing standard error of the mean. Statistical analysis was carried out between the WT and KO macrophages using Welch's $t$ test. ${ }^{*} p<0.05,{ }^{*} p<0.001$. B Time-course analysis shows that MNGC formation occurs earlier and to a greater extent in CD9 null mouse macrophages. C Analysis of early infection of WT and CD9 null
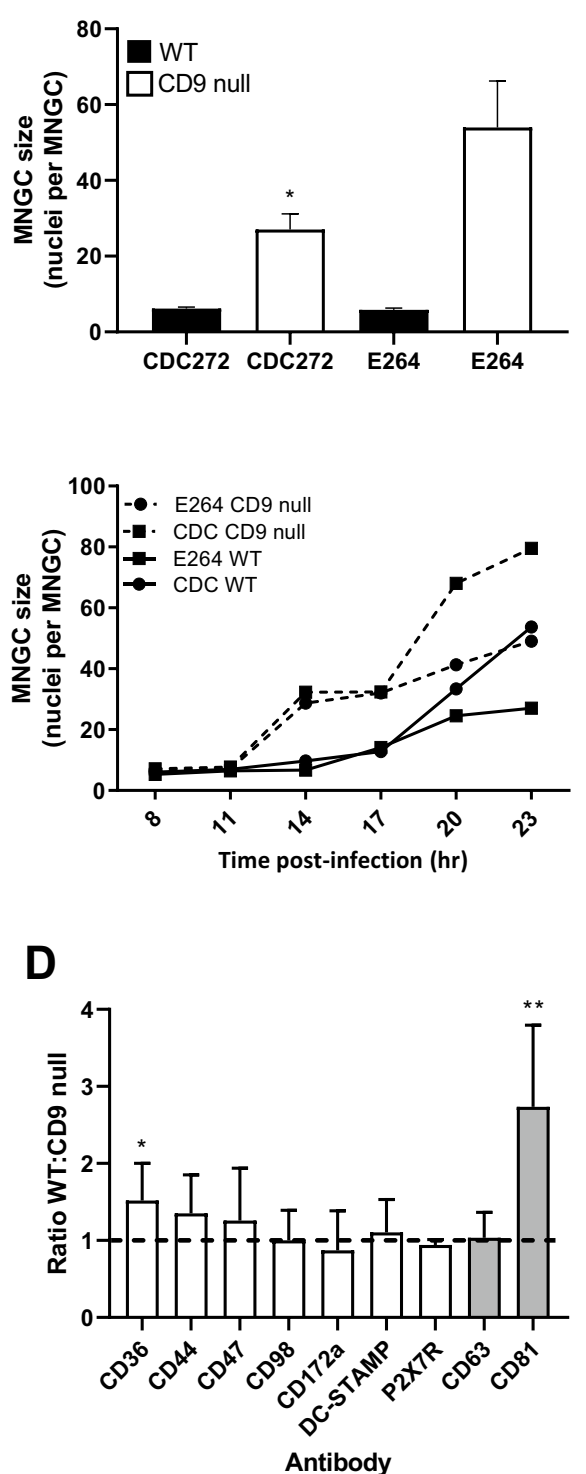

mouse macrophages by $B$. thailandensis CDC272 was performed after the kanamycin protection assay by lysing the macrophages at various time points and determining the number of surviving intracellular bacteria determined (cfu/10,000 host cells). D The surface expression of tetraspanins CD63 and CD81 (grey fill) and non-tetraspanin fusion-related proteins (white fill) was assessed by flow cytometry. RFI values for WT and CD9 null are shown as a ratio, with error bars showing standard error of the mean from at least three independent experiments performed in duplicate. Statistical analysis was assessed by one-sample $t$ test, ${ }^{*} p<0.05, * * p<0.001$ 
A

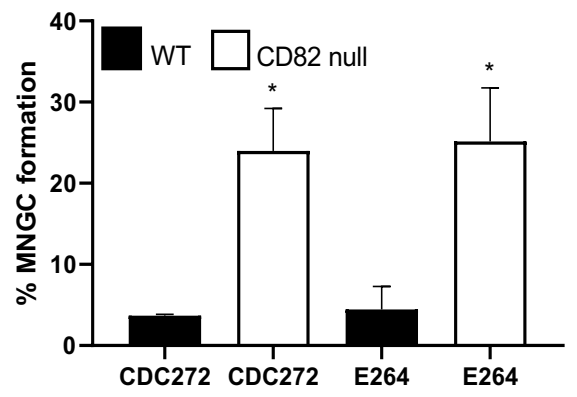

B

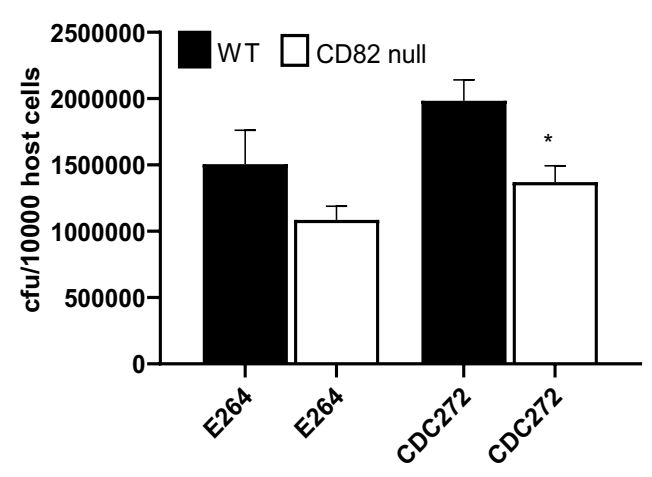

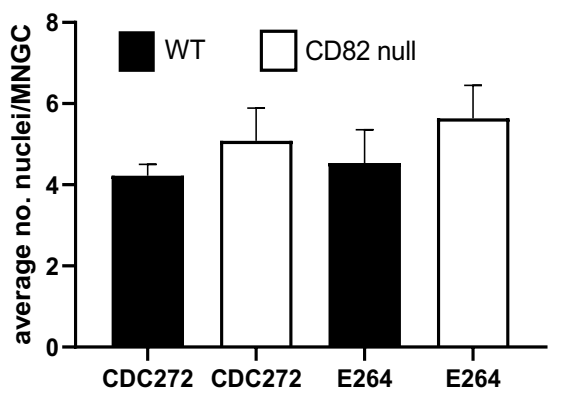

Fig. 7 Effect of CD82 knock-out on MNGC formation induced by $B$. thailandensis strains CDC272 and E264 in mouse macrophages. A Following infection of macrophages derived from WT (black fill) or CD82 null mice (white fill) with both strains of bacteria, MNGC formation was assessed after $14 \mathrm{~h}$, as described previously. The results are representative of three independent experiments, with error bars showing standard deviation of the mean. Statistical analysis was carried out between the WT and KO macrophages using Welch's $t$ test.

Our demonstration that $\mathrm{mAb}$ to this protein significantly inhibit $B$. thailandensis-induced MNGC formation echoes the finding that $\mathrm{mAb}$ to $\mathrm{CD} 47$ suppress $B$. pseudomalleiinduced MNGC formation in human U937 macrophages [13]. DC-STAMP (dendritic cell-specific transmembrane protein) is a seven transmembrane domain protein that is described as critical for osteoclast and foreign-body giant cell formation, with mAb and DC-STAMP knock-out suppressing these processes [35, 49]. Our finding that $\mathrm{mAb}$ to DC-STAMP inhibits $B$. thailandensis-induced MNGC formation in mouse macrophages is consistent with a general role for this protein in controlling mononuclear phagocyte fusion. We also observed some inhibitory effects with antibodies to CD98 (also known as fusion regulatory protein-1 or FRP-1), which is also in line with the anti-CD98 suppression of MNGC formation induced by
${ }^{*} p<0.05,{ }^{* *} p<0.001$. B Analysis of infection of WT and CD82 null mouse macrophages by $B$. thailandensis CDC272 was performed after the kanamycin protection assay by lysing the macrophages $2 \mathrm{~h}$ and determining the number of surviving intracellular bacteria determined (cfu/10,000 host cells). Statistical analysis was carried out between the WT (black fill) and CD82 null (white fill) cells using Welch's $t$ test. ${ }^{*} p<0.05$. Pairs with no asterisk showed no significant difference

B. pseudomallei infection of human U937 macrophages observed by Suparak and co-workers [13]. Interestingly, CD98 is reported to associate with tetraspanins CD9 and $\mathrm{CD} 81$ on the surface of oocytes, where mAb to both CD9 and CD98 inhibited sperm:egg fusion [50].

Taken together, our results demonstrate that tetraspanins CD9 and CD81 are involved in MNGC formation induced by $B$. thailandensis in mouse macrophages, with clear evidence that $\mathrm{CD} 9$ acts as a negative regulator of this process. Given the similarities between their fusion apparatus, in our view, it is highly likely that CD9 also regulates MNGC formation induced by B. pseudomallei. Our findings may, therefore, have implications for the better understanding of this aspect of pathogenesis in melioidosis and could indicate future treatments aimed at controlling bacterial syncytium formation. 
A

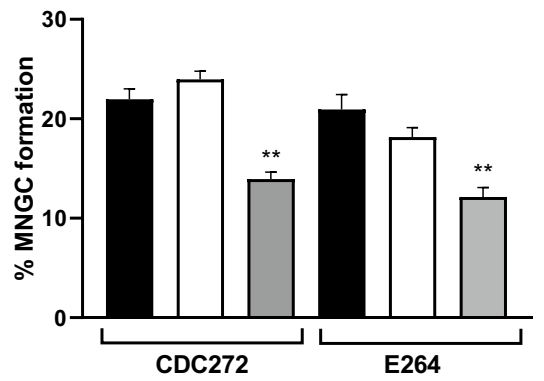

B

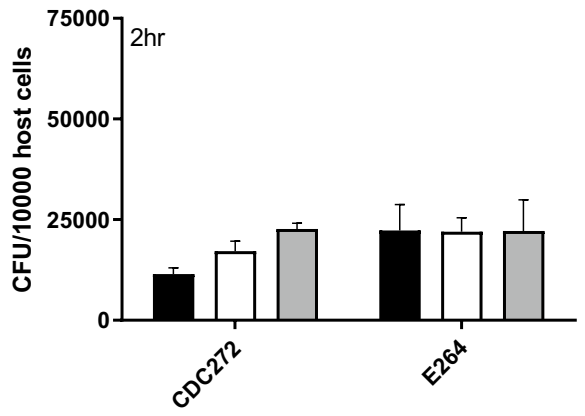

Fig. 8 Effect of CD9 overexpression on MNGC formation induced by B. thailandensis strains CDC272 and E264 in mouse macrophages. A The capacity of $B$. thailandensis strains E264 and CDC272 to induce MNGC formation in WT J774.2 cells (black fill), J774.2 cells stably transfected to overexpress GFP (white fill), or J774.2 cells stably transfected to overexpress mouse CD9-GFP (grey fill) was assessed $16 \mathrm{~h}$ after infection as described previously. Data represent three independent experiments, with error bars showing standard error of the mean. Statistical differences between the cell types were assessed

Acknowledgements This work was supported by PhD scholarships from the Ministry for Higher Education (Libya) and the Ministry of Higher Education and Scientific Research (Iraq). The authors appreciate the skilled technical assistance provided by the Flow Cytometry Unit (Medical School, University of Sheffield) and expert advice provided by Dr Helen Marriott (infection work) in the Dept Infection, Immunity and Cardiovascular Disease. We also thank Drs Marzieh Fanaei, Ibrahim Yaseen, and John Palmer for providing the recombinant EC2 proteins and Jocelyn Pinto for her assistance with work on the CD82 null macrophages (Dept Molecular Biology and Biotechnology, University of Sheffield). We are grateful to Dr. Gabriela Dveksler, Dept. Pathology, Uniformed Services University of Health Sciences, Bethesda, MD, US for providing us with the CD9 null macrophage cell line and to Professor Jatin Vyas, Division of Infectious Disease, Massachusetts General Hospital, Boston, US, for the CD82 null macrophage cell line. We would also like to thank Professor Richard Titbull (Dept. Biosciences, University of Exeter, UK) for providing the B. thailandensis strains used.

\section{Compliance with ethical standards}

Conflict of interest The authors declare that they have no conflict of interest

Consent for publication The authors consent to this work being published.
$\mathrm{J} 774$

J774-GFP

J774-CD9GFP

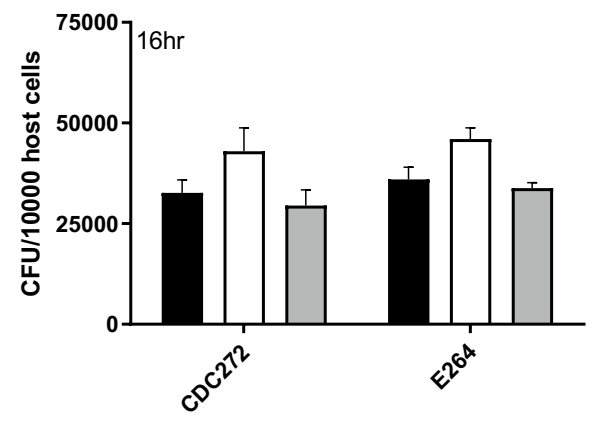

by two-way ANOVA, $* * p<0.001, n s$ non-significant. B Survival of B. thailandensis strains E264 and CDC272 in WT J774.2 cells (black fill), J774. 2 cells stably transfected to overexpress GFP (white fill) or J774.2 cells stably transfected to overexpress mouse CD9-GFP (grey fill). Bacterial survival was assessed at $2 \mathrm{~h}$ and $16 \mathrm{~h}$ after infection by lysing the cells and determining the number of live intracellular bacteria as described previously. The data were analysed as for (A), but no statistical differences between the WT or transfected J774.2 cells were found

Ethical approval Not applicable.

Open Access This article is licensed under a Creative Commons Attribution 4.0 International License, which permits use, sharing, adaptation, distribution and reproduction in any medium or format, as long as you give appropriate credit to the original author(s) and the source, provide a link to the Creative Commons licence, and indicate if changes were made. The images or other third party material in this article are included in the article's Creative Commons licence, unless indicated otherwise in a credit line to the material. If material is not included in the article's Creative Commons licence and your intended use is not permitted by statutory regulation or exceeds the permitted use, you will need to obtain permission directly from the copyright holder. To view a copy of this licence, visit http://creativecommons.org/licenses/by/4.0/.

\section{References}

1. Dance DA et al (1989) Identification of Pseudomonas pseudomalle $i$ in clinical practice: use of simple screening tests and API 20NE. J Clin Pathol 42(6):645-648

2. Wiersinga WJ et al (2018) Melioidosis. Nat Rev Dis Primers 4:17107 
3. Limmathurotsakul D et al (2012) Melioidosis in animals, Thailand, 2006-2010. Emerg Infect Dis 18(2):325-327

4. Ngauy V et al (2005) Cutaneous melioidosis in a man who was taken as a prisoner of war by the Japanese during World War II. J Clin Microbiol 43(2):970-972

5. Stevens JM et al (2005) Actin-binding proteins from Burkholderia mallei and Burkholderia thailandensis can functionally compensate for the actin-based motility defect of a Burkholderia pseudomallei bimA mutant. J Bacteriol 187(22):7857-7862

6. Benanti EL, Nguyen CM, Welch MD (2015) Virulent Burkholderia species mimic host actin polymerases to drive actinbased motility. Cell 161(2):348-360

7. Kespichayawattana $\mathrm{W}$ et al (2000) Burkholderia pseudomallei induces cell fusion and actin-associated membrane protrusion: a possible mechanism for cell-to-cell spreading. Infect Immun 68(9):5377-5384

8. Wong KT, Puthucheary SD, Vadivelu J (1995) The histopathology of human melioidosis. Histopathology 26(1):51-55

9. Harley VS et al (1998) Effects of Burkholderia pseudomallei and other Burkholderia species on eukaryotic cells in tissue culture. Microbios 96(384):71-93

10. Wand ME et al (2011) Macrophage and Galleria mellonella infection models reflect the virulence of naturally occurring isolates of B. pseudomallei, B. thailandensis and B. oklahomensis. BMC Microbiol 11(1):11

11. Schwarz S et al (2014) VgrG-5 is a Burkholderia type VI secretion system-exported protein required for multinucleated giant cell formation and virulence. Infect Immun 82(4):1445-1452

12. Toesca IJ, French CT, Miller JF (2014) The Type VI secretion system spike protein VgrG5 mediates membrane fusion during intercellular spread by pseudomallei group Burkholderia species. Infect Immun 82(4):1436-1444

13. Suparak $\mathrm{S}$ et al (2011) Burkholderia pseudomallei-induced cell fusion in U937 macrophages can be inhibited by monoclonal antibodies against host cell surface molecules. Microbes Infect 13(12-13):1006-1011

14. Charrin $\mathrm{S}$ et al (2014) Tetraspanins at a glance. J Cell Sci 127(Pt 17):3641-3648

15. Yanez-Mo M et al (2009) Tetraspanin-enriched microdomains: a functional unit in cell plasma membranes. Trends Cell Biol 19(9):434-446

16. Evans JP (2012) Sperm-egg interaction. Annu Rev Physiol 74:477-502

17. Rubinstein E et al (2006) The molecular players of sperm-egg fusion in mammals. Semin Cell Dev Biol 17(2):254-263

18. Charrin $\mathrm{S}$ et al (2013) Normal muscle regeneration requires tight control of muscle cell fusion by tetraspanins CD9 and CD81. Nat Commun 4:1674

19. Ishii $\mathrm{M}$ et al (2006) RANKL-induced expression of tetraspanin CD9 in lipid raft membrane microdomain is essential for cell fusion during osteoclastogenesis. J Bone Miner Res 21(6):965-976

20. Takeda $\mathrm{Y}$ et al (2003) Tetraspanins CD9 and CD81 function to prevent the fusion of mononuclear phagocytes. J Cell Biol 161(5):945-956

21. Parthasarathy V et al (2009) Distinct roles for tetraspanins CD9, CD63 and CD81 in the formation of multinucleated giant cells. Immunology 127(2):237-248

22. Hulme RS et al (2014) Distinct regions of the large extracellular domain of tetraspanin CD9 are involved in the control of human multinucleated giant cell formation. PLoS One 9(12):e116289

23. Ho SH et al (2006) Recombinant extracellular domains of tetraspanin proteins are potent inhibitors of the infection of macrophages by human immunodeficiency virus type 1 . J Virol 80(13):6487-6496
24. Monk PN, Partridge LJ (2012) Tetraspanins: gateways for infection. Infect Disord Drug Targets 12(1):4-17

25. Symeonides $\mathrm{M}$ et al (2014) Evidence showing that tetraspanins inhibit HIV-1-induced cell-cell fusion at a post-hemifusion stage. Viruses 6(3):1078-1090

26. Lennings J et al (2019) Environmental and cellular factors affecting the localization of T6SS proteins in Burkholderia thailandensis. Int J Med Microbiol 309(6):151335

27. Yu Y et al (2006) Genomic patterns of pathogen evolution revealed by comparison of Burkholderia pseudomallei, the causative agent of melioidosis, to avirulent Burkholderia thailandensis. BMC Microbiol 6:46

28. Glass MB et al (2006) Pneumonia and septicemia caused by Burkholderia thailandensis in the United States. J Clin Microbiol 44(12):4601-4604

29. Le Naour $F$ et al (2000) Severely reduced female fertility in CD9deficient mice. Science 287(5451):319-321

30. Ha CT et al (2005) Binding of pregnancy-specific glycoprotein 17 to CD9 on macrophages induces secretion of IL-10, IL-6, PGE2, and TGF-beta1. J Leukoc Biol 77(6):948-957

31. Risinger JI et al (2014) Normal viability of Kai1/Cd82 deficient mice. Mol Carcinog 53(8):610-624

32. Brett PJ, DeShazer D, Woods DE (1998) Burkholderia thailandensis sp. nov., a Burkholderia pseudomallei-like species. Int J Syst Bacteriol 48(Pt 1):317-320

33. Higginbottom A et al (2003) Structural requirements for the inhibitory action of the CD9 large extracellular domain in sperm/oocyte binding and fusion. Biochem Biophys Res Commun 311(1):208-214

34. Caplan MJ, Kamsteeg EJ, Duffield A (2007) Tetraspan proteins: regulators of renal structure and function. Curr Opin Nephrol Hypertens 16(4):353-358

35. Yagi $M$ et al (2005) DC-STAMP is essential for cell-cell fusion in osteoclasts and foreign body giant cells. J Exp Med 202(3):345-351

36. Boddey JA et al (2007) The bacterial gene lfpA influences the potent induction of calcitonin receptor and osteoclast-related genes in Burkholderia pseudomallei-induced TRAP-positive multinucleated giant cells. Cell Microbiol 9(2):514-531

37. Higginbottom A et al (2000) Antibody cross-linking of human CD9 and the high-affinity immunoglobulin E receptor stimulates secretion from transfected rat basophilic leukaemia cells. Immunology 99(4):546-552

38. Barreiro $\mathrm{O}$ et al (2005) Endothelial tetraspanin microdomains regulate leukocyte firm adhesion during extravasation. Blood 105(7):2852-2861

39. Helming L, Gordon S (2009) Molecular mediators of macrophage fusion. Trends Cell Biol 19(10):514-522

40. Helming L, Winter J, Gordon S (2009) The scavenger receptor CD36 plays a role in cytokine-induced macrophage fusion. J Cell Sci 122(Pt 4):453-459

41. Fanaei M, Monk PN, Partridge LJ (2011) The role of tetraspanins in fusion. Biochem Soc Trans 39(2):524-528

42. Park SY, Yun Y, Kim IS (2012) CD36 is required for myoblast fusion during myogenic differentiation. Biochem Biophys Res Commun 427(4):705-710

43. Huang W, Febbraio M, Silverstein RL (2011) CD9 tetraspanin interacts with $\mathrm{CD} 36$ on the surface of macrophages: a possible regulatory influence on uptake of oxidized low density lipoprotein. PLoS One 6(12):e29092

44. Kania JR, Kehat-Stadler T, Kupfer SR (1997) CD44 antibodies inhibit osteoclast formation. J Bone Miner Res 12(8):1155-1164

45. Sterling H, Saginario C, Vignery A (1998) CD44 occupancy prevents macrophage multinucleation. J Cell Biol 143(3):837-847

46. de Vries TJ et al (2005) Effect of CD44 deficiency on in vitro and in vivo osteoclast formation. J Cell Biochem 94(5):954-966 
47. Le Naour F et al (2006) Profiling of the tetraspanin web of human colon cancer cells. Mol Cell Proteomics 5(5):845-857

48. Han X et al (2000) CD47, a ligand for the macrophage fusion receptor, participates in macrophage multinucleation. J Biol Chem 275(48):37984-37992

49. Kukita T et al (2004) RANKL-induced DC-STAMP is essential for osteoclastogenesis. J Exp Med 200(7):941-946
50. Takahashi Y et al (2001) Sequence-specific interaction between the disintegrin domain of mouse ADAM 3 and murine eggs: role of beta1 integrin-associated proteins CD9, CD81, and CD98. Mol Biol Cell 12(4):809-820

Publisher's Note Springer Nature remains neutral with regard to jurisdictional claims in published maps and institutional affiliations. 